\title{
AVALIAÇÃO SENSORIAL DE REQUEIJÃO CREMOSO DE DIFERENTES MARCAS COMERCIAIS
}

\section{SENSORY ASSESSMENT OF CREAMY CHEESE OF DIFFERENT TRADEMARKS}

\author{
F. R. PASSOS ${ }^{1}$, W. D. B. ARAÚJO ${ }^{1}$, L. RIBEIRO ${ }^{1}$, R. A. R. TEODORO ${ }^{1}$, R. V. B. \\ FERNANDES ${ }^{1}$, F. Q.MENDES ${ }^{1}$
}

Universidade Federal de Viçosa

Campus Rio Paranaíba

E-mail: flaviapassos1@yahoo.com.br

\author{
article info \\ Article history: \\ Received 12 May 2017 \\ Accepted 3 August 2017 \\ Available online 20 September 2017
}

PALAVRAS-CHAVE: Queijo fundido; Análise sensorial; Influência do sexo.

KEYWORDS: Processed cheese; Sensory analysis; Influence of sex.

RESUMO: $O$ requeijão cremoso é um tipo de queijo fundido originalmente brasileiro, fabricado em todo território nacional e é um dos principais tipos de queijos produzido e consumido no Brasil. O presente trabalho teve como objetivo avaliar sensorialmente cinco marcas comerciais de requeijão cremoso. As amostras de requeijão foram codificadas com as letras $A, B, C, D$ e $E$ e submetidas à análise sensorial por uma equipe de 54 provadores não treinados de ambos os sexos. Os atributos aparência, cor, consistência, sabor e impressão global foram avaliados através da escala hedônica de 9 pontos. Os resultados foram submetidos à Análise de Variância (ANOVA), seguida pelo teste de Tukey, ao nível de 5\% de significância. O requeijão da marca $C$ obteve menores médias, diferindo estatisticamente $(p<0,05)$ em todos os atributos das demais amostras. Em relação à influência do sexo, a amostra $C$ foi a menos aceita pelo público feminino. Para o público masculino, a amostra $C$ também foi menos aceita, porém não houve diferença significativa $(p>0,05)$ com todas as amostras analisadas em relação à cor, com as amostras $B$ e $C$ em relação à aparência, sabor e impressão global, e consistência entre as amostras $B, C$ e D.

\begin{abstract}
Cream cheese is a type of originally brazilian cheese, manufactured throughout the country and is one of the main types of cheeses produced and consumed in Brazil. The objective of this study was to evaluate sensorially five commercial brands of cream cheese. The curd samples were coded with letters $A, B, C, D$ and $E$ and submitted to sensory analysis by a team of 54 untrained tasters of both sexes. The attributes appearance, color, consistency, taste and overall impression were evaluated through the hedonic scale of 9 points. The results were submitted to Analysis of Variance (ANOVA), followed by the Tukey test, at the 5\% level of significance. The $C$ brand curd had lower mean values, differing statistically $(p<0.05)$ in all attributes of the other samples. Regarding the influence of gender, sample $C$ was the least accepted by the female audience. For the male audience, sample $C$ was also less accepted, but there was no significant difference ( $p>0.05)$ with all samples analyzed in relation to color, with samples $B$ and $C$ in relation to appearance, taste and overall impression, and consistency between samples $B, C$ and $D$.
\end{abstract}

\section{INTRODUÇÃO}

A produção de queijos fundidos difundiu-se em vários países, ganhando grande destaque nos laticínios (BAYARRI et al., 2012). No Brasil, a produção e o consumo de 


\section{The Journal of Engineering and Exact Sciences - \\ JCEC \\ ISSN: 2527-1075}

queijos processados aumentou consideravelmente, com destaque para o requeijão cremoso considerado um tipo de queijo fundido (OLIVEIRA et al., 2015).

Segundo a legislação brasileira, o requeijão cremoso é obtido por fusão da massa coalhada, cozida ou não, dessorada e lavada, obtida por coagulação ácida e/ou enzimática do leite, opcionalmente adicionada de creme de leite e/ou manteiga e/ou gordura anidra de leite e/ou butter oil. O mais comum é aquele fabricado por acidificação do leite com ácido lático a quente (BRASIL, 1997).

As características sensoriais do requeijão cremoso, como sabor, aroma, aparência e textura são primordiais para provocar aceitação positiva do mesmo. Alguns dos defeitos mais comuns em requeijão cremoso são: consistência granulada; fluidez heterogênea; fluidez excessiva; emulsão brilhosa ou com separação de gordura; descoloração ou escurecimento do produto; textura arenosa, cristalizada; defeitos de sabor, como sabor fraco, picante, amargo, rançoso, ácido, químico, alcalino e metálico (GARRUTI et al., 2003). Existem poucos trabalhos científicos disponíveis na literatura, abordando a qualidade sensorial do produto comercializado. Assim, torna-se necessário, avaliar as diferentes marcas de requeijão cremoso disponíveis no mercado. Dessa forma, o objetivo desse trabalho foi avaliar a aceitação sensorial de requeijão cremoso de cinco marcas comercializados na região Alto Paranaíba de Minas Gerais.

\section{MATERIAL E MÉTODOS}

Foram avaliadas cinco marcas comerciais de requeijão envasados em copos plásticos de $250 \mathrm{~mL}$, adquiridos em supermercados do município de Patos de Minas - MG. A análise sensorial foi realizada na Universidade Federal de Viçosa Campus Rio Paranaíba (UFV/CRP).

Os provadores, constituídos por estudantes, professores e funcionários da UFV/CRP, com idade entre 18 e 61 anos foram selecionados quanto ao hábito de consumir requeijão cremoso e em função da disponibilidade e do interesse em participar do teste. As amostras de requeijão foram codificadas com as letras A, B, C, D e E e submetidas à análise sensorial por uma equipe de 54 provadores não treinados de ambos os sexos, sendo $60 \%$ mulheres e $40 \%$ homens. Cada provador recebeu um copo de requeijão de cada vez, codificado com número de três dígitos aleatórios (REIS e MINIM, 2010), sendo-lhe solicitado manusear o produto com uma faca, espalhar sobre um biscoito tipo água e sal (GARRUTI et al., 2003), avaliar a amostra de uma forma geral (impressão global) e, em seguida, quanto à aparência, cor, consistência e sabor. Esses atributos foram avaliados através da escala hedônica de nove pontos, variando de uma extremidade a outra desde "gostei extremamente" com nota igual a 9 à "desgostei extremamente" com nota igual a 1, segundo metodologia descrita por Dutcosky (2013).

Os resultados foram submetidos à Análise de Variância (ANOVA), seguida pelo teste de Tukey, ao nível de 5\% de significância para comparação entre as médias, utilizando-se o Programa Estatístico SPSS Statistics 17.0. 


\section{The Journal of Engineering and Exact Sciences - \\ JCEC}

ISSN: 2527-1075

\section{RESULTADOS E DISCUSSÃO}

As médias das notas atribuídas pelos provadores a cada atributo, para cada uma das marcas de requeijão cremoso avaliadas sensorialmente, são apresentados na Tabela 1 .

Tabela 1 - Médias dos valores atribuídos pelos provadores a cada atributo para as amostras de requeijão cremoso

\begin{tabular}{cccccc}
\hline \multirow{2}{*}{ Amostras } & \multicolumn{5}{c}{ Atributos } \\
\cline { 2 - 6 } & Aparência & Cor & Consistência & Sabor & $\begin{array}{c}\text { Impressão } \\
\text { global }\end{array}$ \\
\hline $\mathbf{A}$ & $7,59^{\mathrm{a}}$ & $7,94^{\mathrm{a}}$ & $7,39^{\mathrm{a}}$ & $6,74^{\mathrm{a}}$ & $7,00^{\mathrm{a}}$ \\
$\mathbf{B}$ & $7,37^{\mathrm{a}}$ & $7,46^{\mathrm{a}}$ & $7,04^{\mathrm{a}}$ & $5,92^{\mathrm{a}}$ & $6,70^{\mathrm{a}}$ \\
$\mathbf{C}$ & $6,07^{\mathrm{b}}$ & $6,68^{\mathrm{b}}$ & $5,05^{\mathrm{b}}$ & $4,02^{\mathrm{b}}$ & $4,94^{\mathrm{b}}$ \\
$\mathbf{D}$ & $7,61^{\mathrm{a}}$ & $7,80^{\mathrm{a}}$ & $7,28^{\mathrm{a}}$ & $6,85^{\mathrm{a}}$ & $7,35^{\mathrm{a}}$ \\
$\mathbf{E}$ & $7,48^{\mathrm{a}}$ & $7,83^{\mathrm{a}}$ & $7,22^{\mathrm{a}}$ & $6,80^{\mathrm{a}}$ & $7,33^{\mathrm{a}}$ \\
\hline
\end{tabular}

a-bMédias na mesma coluna acompanhadas de mesma letra não diferem entre si ao nível de $5 \%$ de significância.

$O$ requeijão da marca $C$ obteve menores médias, diferindo estatisticamente $(p<0,05)$ em todos os atributos das demais amostras. Os valores das médias da amostra $\mathrm{C}$ variaram desde "desgostei ligeiramente" a "gostei moderadamente". As amostras A, B, D e E apresentaram médias entre os termos hedônicos "gostei moderadamente" a "gostei muito" para os atributos aparência, cor, consistência e impressão global, e em relação ao atributo sabor, correspondeu entre os termos hedônicos "gostei ligeiramente" a "gostei moderadamente".

A amostra $\mathrm{C}$ possuía o menor preço e continha amido modificado na sua composição, alterando o sabor (não característico de requeijão, sabor fraco), consistência elevada, pouco lisa e coloração escura. Produtos que contém amido modificado, gordura vegetal e concentrado protéico do soro são designados "especialidades lácteas à base de requeijão", a qual tem menor custo de produção do que a do requeijão cremoso, pois há substituição do leite por produtos que aumentam o rendimento. Além dos ingredientes serem outros, as especialidades lácteas possuem valores de umidade e matéria gorda no extrato seco diferentes das do requeijão. A umidade é maior, e a matéria gorda, menor. As especialidades lácteas não podem trazer escrito "requeijão" no rótulo, e sim, devem constar apenas termos como "cremoso" e "tradicional" (INMETRO, 2012). Com esta afirmativa, pode-se observar que a rotulagem do requeijão cremoso da marca $\mathrm{C}$, não atende aos parâmetros descritos na legislação.

Silva et al. (2006) defendem que requeijão cremoso e especialidade láctea a base de requeijão devem ser vendidos separadamente no supermercado, para não confundir os consumidores. Boa parte das especialidades lácteas é confundida com requeijão porque as empresas estampam o verdadeiro tipo de produto em letras miúdas no rótulo e/ou nem trazem escrito o nome real do produto (INMETRO, 2012), conforme encontrado na pesquisa. Assim, o consumidor menos atento acaba não percebendo que está diante de produtos diferentes e que deveriam ter preços também distintos. 


\section{The Journal of Engineering and Exact Sciences - \\ JCEC \\ ISSN: 2527-1075}

Na Tabela 2 é apresentada as médias dos atributos analisados em relação à influência do sexo na aceitação das amostras de requeijão cremoso.

Tabela 2 - Médias dos valores atribuídos pelos provadores, segundo o sexo, a cada atributo, para as amostras de requeijão cremoso

\begin{tabular}{ccccccccccc}
\hline Amostra & \multicolumn{2}{c}{ Aparência } & \multicolumn{2}{c}{ Cor } & \multicolumn{2}{c}{ Consistência } & Sabor & \multicolumn{2}{c}{$\begin{array}{c}\text { Impressão } \\
\text { global }\end{array}$} \\
& & & & & & & & & & \\
\hline & $\mathbf{M}$ & $\mathbf{F}$ & $\mathbf{M}$ & $\mathbf{F}$ & $\mathbf{M}$ & $\mathbf{F}$ & $\mathbf{M}$ & $\mathbf{F}$ & $\mathbf{M}$ & $\mathbf{F}$ \\
\hline & $7,53^{\mathrm{a}}$ & $7,61^{\mathrm{a}}$ & $8,00^{\mathrm{a}}$ & $7,92^{\mathrm{a}}$ & $7,20^{\mathrm{a}}$ & $7,46^{\mathrm{a}}$ & $7,00^{\mathrm{a}}$ & $6,64^{\mathrm{a}}$ & $7,07^{\mathrm{a}}$ & $6,97^{\mathrm{a}}$ \\
$\mathbf{B}$ & $7,20^{\mathrm{ab}}$ & $7,43^{\mathrm{a}}$ & $7,27^{\mathrm{a}}$ & $7,54^{\mathrm{a}}$ & $6,93^{\mathrm{ab}}$ & $7,08^{\mathrm{a}}$ & $6,13^{\mathrm{ab}}$ & $5,85^{\mathrm{a}}$ & $6,40^{\mathrm{ab}}$ & $6,82^{\mathrm{a}}$ \\
$\mathbf{C}$ & $6,13^{\mathrm{b}}$ & $6,05^{\mathrm{b}}$ & $7,00^{\mathrm{a}}$ & $6,56^{\mathrm{b}}$ & $5,20^{\mathrm{b}}$ & $5,00^{\mathrm{b}}$ & $4,93^{\mathrm{b}}$ & $3,67^{\mathrm{b}}$ & $5,40^{\mathrm{b}}$ & $4,77^{\mathrm{b}}$ \\
$\mathbf{D}$ & $7,47^{\mathrm{a}}$ & $7,67^{\mathrm{a}}$ & $7,73^{\mathrm{a}}$ & $7,82^{\mathrm{a}}$ & $6,93^{\mathrm{ab}}$ & $7,41^{\mathrm{a}}$ & $7,07^{\mathrm{a}}$ & $6,77^{\mathrm{a}}$ & $7,07^{\mathrm{a}}$ & $7,46^{\mathrm{a}}$ \\
$\mathbf{E}$ & $7,40^{\mathrm{a}}$ & $7,51^{\mathrm{a}}$ & $8,00^{\mathrm{a}}$ & $7,77^{\mathrm{a}}$ & $7,13^{\mathrm{a}}$ & $7,26^{\mathrm{a}}$ & $7,13^{\mathrm{a}}$ & $6,67^{\mathrm{a}}$ & $7,53^{\mathrm{a}}$ & $7,26^{\mathrm{a}}$ \\
\hline
\end{tabular}

a-b: Médias na mesma coluna acompanhadas de mesma letra não diferem entre si ao nível de $5 \%$ de significância.

M: Masculino

F: Feminino

A amostra $\mathrm{C}$ diferiu $(\mathrm{p}<0,05)$ das demais amostras em todos os atributos, em relação ao sexo feminino, obtendo menores médias, correspondendo entre os termos hedônicos "desgostei ligeiramente" a "desgostei moderadamente".

Em relação ao sexo masculino, o atributo aparência não diferiu significativamente ( $p>0,05)$ entre as amostras B e C, ficando entre os termos hedônicos "gostei ligeiramente" e "gostei moderadamente", respectivamente; porém a amostra $C$ se diferiu $(p<0,05)$ das amostras A, D e E, correspondendo entre os termos "gostei moderadamente" e "gostei muito". No quesito cor, as amostras não se diferiram entre si ( $p>0,05)$, com valores médios entre os termos hedônicos "gostei moderadamente" e "gostei muito". Quanto ao atributo consistência, as amostras B, C e D não se diferiram entre si ( $>0,05)$, uma vez que na escala hedônica estava entre os termos "indiferente" a "gostei moderadamente", porém a amostra C se diferiu $(p<0,05)$ das amostras A e E. Analisando o atributo sabor e impressão global, não foi verificada diferença significativa $(p>0,05)$ entre as amostras $B$ e $C$, porém a amostra $C$ se diferiu $(p<0,05)$ das amostras $A, D$ e $E$, com valores médios próximos a "gostei moderadamente", enquanto a amostra $\mathrm{C}$ apresentou média próxima do termo hedônico "indiferente".

\section{CONCLUSÕES}

A amostra $\mathrm{C}$ é a menos aceita entre os provadores para todos os atributos avaliados. Ambos os sexos se comportam de forma distinta. O público feminino verifica diferença apenas na amostra $\mathrm{C}$ e semelhanças entre as demais amostras em relação aos atributos avaliados. O público masculino verifica semelhanças entre as amostras $\mathrm{B}$ e $\mathrm{C}$ em relação aos atributos aparência, sabor e impressão global, nenhuma diferença significativa quanto à cor e consistência, com semelhança entre as amostras B, C e D. 
É necessária fiscalização mais eficiente pelos órgãos competentes a fim de que a designação do produto esteja informada de forma adequada nos rótulos, conforme estabelecido por lei.

\section{REFERÊNCIAS}

BAYARRI, S.; CARBONELL, I.; COSTELL, E. Viscoelasticity and texture of spreadable cheeses with different fat contents at refrigeration and room temperatures. Journal of Dairy Science, Champaign, v. 95, n. 12, p. 6926-6936, 2012.

BRASIL. Ministério da Agricultura, Pecuária e Abastecimento. Portaria no 359, de 04 de setembro de 1997 (D.O.U 08/09/97). Aprova o Regulamento Técnico para Fixação de Identidade e Qualidade de Requeijão ou Requesón. Diário Oficial [da] República Federativa do Brasil, Poder Executivo, Brasília, Seção 1, p. 19690, 1997.

DUTCOSKY, S. D. Análise sensorial de alimentos. 4. ed. Curitiba: Champagnat, 2013. 531 p.

GARRUTI, D. S.; BRITO, E. S.; BRANDÃO, T. M.; UCHÔA JR., P.; SILVA, M. A. A. P. Desenvolvimento do perfil sensorial e aceitação do requeijão cremoso. Revista Ciência e Tecnologia de Alimentos, Campinas, v. 23, n. 3, p. 434-440, 2003.

INMETRO. Instituto Nacional de Metrologia, Qualidade e Tecnologia. Requeijão e especialidade láctea à base de requeijão, 2012. Disponível em:

$<$ http://www.inmetro.gov.br/consumidor/produtos/requeijao.asp>. Acesso em: 25 jan. 2017.

OLIVEIRA, B. R.; ALMEIDA, C. C.; MEIRA, W. M.; FREITAS, M. Q. Análise sensorial de requeijão cremoso tradicional e "light" reduzido de sódio e gordura. Enciclopédia Biosfera, Goiânia, v. 11, n. 21, p. 152-158, 2015.

REIS, R. C.; MINIM, V. P. R. Teste de aceitação. In: MINIM, V. P. R. Análise sensorial: estudos com consumidores. 2. ed. Viçosa: Ed. UFV, 2010. Cap.3, p.66-82.

SILVA, G. O.; TAKIZAWA, F. F.; PEDROSO, R. A.; FRANCO, C. M. L.; LEONEL, M.; SARMENTO, S. B. S.; DEMIATE, I. M. Características físico-químicas de amidos modificados de grau alimentício comercializados no Brasil. Ciência e Tecnologia de Alimentos, Campinas, v. 26, n. 1, p. 188-197, 2006. 